\title{
A case report of short-chain acyl-CoA dehydrogenase deficiency (SCADD)
}

\author{
Barbka Repic Lampret', Simona Murk01, Marusa Debeljak ${ }^{1}$ Mojca Zerjav Tansek1, Petja Fister ${ }^{1}$, Tadej Battelino*1,2 \\ 1University Medical Centre Ljubljana, University Children's Hospital, Ljubljana, Slovenia \\ 2University of Ljubljana, Faculty of Medicine, Department of Paediatrics, Ljubljana, Slovenia \\ *Corresponding author: tadej.battelino@mf.uni-lj.si
}

\begin{abstract}
Background: Short-chain acyl-COA dehydrogenase deficiency (SCADD) is a rare inherited mitochondrial fatty acid oxidation disorder associated with variations in the ACADS (Acyl-CoA dehydrogenase, $(-2$ to $C-3$ short chain) gene. SCADD has highly variable biochemical, genetic and clinical characteristics. Phenotypes vary from fatal metabolic decompensation to asymptomatic individuals.
\end{abstract}

Subject and methods: A Romani boy presented at 3 days after birth with hypoglycaemia, hypotonia and respiratory pauses with brief generalized seizures. Afterwards the failure to thrive and developmental delay were present. Organic acids analysis with gas chromatography-mass spectrometry (GS/MS) in urine and acylcarnitines analysis with liquid chromatography-tandem mass spectrometry (LC-MS/MS) in dried blood spot were measured. Deoxyribonucleic acid (DNA) was isolated from blood and polymerase chain reactions (PCRs) were performed for all exons. Sequence analysis of all exons and flanking intron sequences of ACADS gene was performed.

Results: Organic acids analysis revealed increased concentration of ethylmalonic acid. Acylcarnitines analysis showed increase of butyrylcarnitine, (4-carnitine. (4-carnitine was 3.5 times above the reference range $(<0.68 \mu \mathrm{mol} / \mathrm{L})$. Confirmation analysis for organic acids and acylcarnitine profile was performed on the second independent sample and showed the same pattern of increased metabolites. Sequence analysis revealed 3-bp deletion at position 310-312 in homozygous state (c.310_312delGAG). Mutation was previously described as pathogenic in heterozygous state, while it is in homozygous state in our patient.

Conclusions: In our case clinical features of a patient, biochemical parameters and genetic data were consistent and showed definitely SCAD deficiency.

Key words: SCAD deficiency; short chain acyl-CoA dehydrogenase deficiency; screening; acylcarnitine; polymorphism, genetic

\section{Introduction}

Short-chain acyl-coenzyme A dehydrogenase deficiency (SCADD) is an autosomal recessive inborn error of mitochondrial fatty acid disorder (FAO) (13). Birth prevalence of SCADD from newborn screening in California was reported to be 1 in 34,632 (4). Similar data was reported from the New England newborn screening program, approximately 1 in 33,000 (5). Birth prevalence of SCADD with a mutation/variant genotype in the Netherlands as high as $>1: 1000$ was calculated. This is in contrast with lower number of patients diagnosed clinically (6).
SCAD is encoded by the ACADS (Acyl-CoA dehydrogenase, $C-2$ to $C-3$ short chain) gene, and a number of mutations have been associated with deficient enzyme activity (7-10). The ACADS gene maps to the 12-chromosome (12q24) and spans 14 $\mathrm{kb}$ of genomic deoxyribonucleic acid (DNA), its 1.9 $\mathrm{kb}$ coding region consists of 10 exons (7). SCAD is the first enzyme of the mitochondrial short-chain $\beta$-oxidation spiral catalyzing the dehydrogenation of $C 4$ and $C 6$ fatty acids (7). Impaired SCAD activity results in accumulation of butyrylcarnitine (C4-car- 
nitine), butyrylglycine, ethylmalonic acid and methylsuccinic acid in blood and urine (4). C4-carnitine, measured in blood, and ethylmalonic acid, measured in urine, are generally used as biochemical markers for SCADD, although accumulation of four-carbon carnitine esters, C4-carnitine, occurs in two other inherited conditions: isobutyryl-CoA dehydrogenase (IBD) deficiency (11) and ethylmalonic encephalopathy (12).

The first two cases of SCADD were reported in 1987 in two neonates who excreted increased concentration of urinary ethylmalonate (5). SCADD is a heterogeneous condition that has been associated with various clinical phenotypes ranging from fatal metabolic decompensation in infancy to asymptomatic individuals (13). Clinical symptoms include development delay, hypotonia, epilepsy, behavioural disorders and hypoglycaemia (3). Most SCADD patients have been diagnosed as a result of investigations for neurological symptoms and/or hypoglycaemia $(1,10,14,15)$. However, many asymptomatic patients have been diagnosed through newborn screening programs.

In this report, we present a first case of SCADD diagnosed in Slovenia, with until now the second described deletion in ACADS gene. Genetic analysis is in accordance with biochemical findings and clinical picture of the described patient.

\section{Patient and methods}

\section{Case history}

The Romani child was born to no consanguineous parents after uneventful pregnancy by spontaneous delivery at 36 weeks of gestation with birth weight of $2550 \mathrm{~g}$ (25-50th percentile), birth length $46 \mathrm{~cm}$ (10-50th percentile), head circumference 33 $\mathrm{cm}$ (50th percentile) and Apgar scores 1 and $5 \mathrm{~min}$ utes after birth of 9/9, respectively. On the first day of life low blood glucose concentration (1.9 $\mathrm{mmol} / \mathrm{L}$ ) was measured. Parenteral infusion of $10 \%$ glucose solution was performed to maintain normal blood glucose concentration. Three days after birth hypotonia and respiratory pauses with brief generalized seizures occurred. At 4 months of age the failure to thrive and moderate developmental delay with muscular hypertrophy were noticed. Informed consent for genetic testing was obtained from parents and all analyses were performed as a part of diagnostic procedure according to the principles of the Helsinki Declaration.

\section{Materials}

Random urine sample without addition of preservative was collected for organic acids analysis, creatinine was measured and urine was stored at $-20{ }^{\circ} \mathrm{C}$ prior the analysis. Analysis was done within 3 days. For the determination of acylcarnitines capillary blood was spotted directly on Whatman 903 filter paper (Whatman GmbH, Dassel, Germany). Blood samples were allowed to dry at room temperature for at least 4 hours. They were stored at $8{ }^{\circ} \mathrm{C}$ prior the analysis and analysis was done within 3 days. Urine samples and dried blood spots were collected at $6^{\text {th }}$ day after birth (sampling I) and again at $13^{\text {th }}$ day after birth (sampling II). Peripheral venous blood sample for DNA isolation $(6 \mathrm{~mL})$ was obtained by venipuncture into EDTA tubes (Becton Dickinson, Milan, Italy).

\section{Biochemical methodology}

Creatinine was determined by Jaffe reaction on Beckman Coulter AU 400 analyzer (Beckman Coulter, Brea, CA, USA). Qualitative determination of organic acids was performed on gas chromatography-mass spectrometry (GC/MS) system (Agilent Technologies, Wilmington, USA). Urine sample was oximated with hydroxyl-amine (O-Ethylhydroxylamine hydrochloride, Sigma-Aldrich, Steinheim, Germany). An internal standard, 2-phenylbutyric acid (Sigma-Aldrich, Steinheim, Germany), was added in concentration of $100 \mathrm{mmol} / \mathrm{mol}$ creatinine. Urine was acidified with $\mathrm{HCl}$ (Riedel-de Haën, Sigma-Aldrich, Seelze, Germany), saturated with $\mathrm{NaCl}$ (Sigma-Aldrich, Steinheim, Germany) and organic acids were extracted using ethyl-acetate (Sigma-Aldrich, Steinheim, Germany). Ethyl-acetate layer was separated and evaporated under the steam of nitrogen. The dried residue was dissolved in pyridine (Sigma-Aldrich, Steinheim, Germany), derivatised with BSTFA (N,O-bis(trimethylsily) trifluoroacetamide, Sigma-Aldrich, Steinheim, Ger- 
many) and injected into GC/MS system. Chromatographic conditions were as followed: initial temperature $70{ }^{\circ} \mathrm{C}$, initial time 2 minutes, rate $3.5^{\circ} \mathrm{C} /$ min, final temperature $270^{\circ} \mathrm{C}$, final time 2 minutes. The obtained spectra were compared with the known library spectra (W8N08 library).

Determination of acylcarnitines from filter paper were performed on tandem mass spectrometer 3200Q Trap (ABSCIEX, Singapore) with Chromsystems MassChrom Aminoacid and Acylcarnitines from dried blood spot commercial reagents kit (Grüfelfing, Germany). The sample preparation was based on extraction followed by derivatisation to butyric esters. Positive electro spray ionization and multiple reactions monitoring (MRM) mode were used.

\section{Molecular diagnosis}

Molecular studies were performed on DNA extracted from peripheral blood leukocytes using FlexiGene isolation kit (Qiagene, Hilden, Germany) from patient and his parents. Polymerase chain reaction (PCR) primers (Eurofins MWG Operon, Ebersberg, Germany) were designed on our own according to the established laboratory protocol (Table 1), covering whole coding regions and intron/exon boundaries of ACADS gene. All PCR reactions were performed with GoTaq G2 Flexi DNA polymerase (Promega, Madison, USA) using 100 ng of double stranded DNA, $0.2 \mathrm{mM}$ of each dNTPs (Life Technologies, Carlsbad, USA), $0.32 \mu \mathrm{M}$ of primers, $2 \mathrm{mM}$ of $\mathrm{MgCl}_{2}$ (Promega, Madison, USA), $1 \mathrm{X}$ green buffer (Promega, Madison, USA) and 0.75 $\mathrm{U}$ of polymerase (Promega, Madison, USA) in a final reaction volume of $25 \mu \mathrm{L}$. The thermo cycling procedure consisted of initial denaturing step at $95^{\circ} \mathrm{C}$ for 2 min followed by 35 cycles of $94^{\circ} \mathrm{C}$ for 30 $\mathrm{s}$, annealing step at $58^{\circ} \mathrm{C}$ for $30 \mathrm{~s}$, extension step at $72^{\circ} \mathrm{C}$ for $30 \mathrm{~s}$ and final extension at $72^{\circ} \mathrm{C}$ for $7 \mathrm{~min}$.

Sequence analysis of all exons and flanking intron sequences of ACADS gene was performed using 3500 genetic analyzer (Applied Biosystem, USA). All identified mutations/variants were validated in additional independent round of PCR and once again sequenced.
TABLE 1. Sequences of PCR primers for ACADS gene.

\begin{tabular}{|c|c|}
\hline Primer & Sequence \\
\hline ACADS e1F/R & $\begin{array}{l}\text { gcagtcgagcgtcggttc } \\
\text { caaggagcagcagaactgg }\end{array}$ \\
\hline ACADS e2F/R & $\begin{array}{l}\text { cctccctggtgagttagtgg } \\
\text { tgactgtcactgccaccatt }\end{array}$ \\
\hline ACADS e3F/R & $\begin{array}{l}\text { tcacatggcctgagtttctg } \\
\text { ggcctacccagtaggacca }\end{array}$ \\
\hline ACADS e4F/R & $\begin{array}{c}\text { gtaggccctggacagaacag } \\
\text { gcctagcaccttcctctcct }\end{array}$ \\
\hline ACADS e 5 AF/R & $\begin{array}{l}\text { agctttgggaccctcatctt } \\
\text { ttgccccagagcaaaaatag }\end{array}$ \\
\hline ACADS e5BF/R & $\begin{array}{c}\text { acagagccctgcaaaacaag } \\
\text { ctcagccacaccctcacac }\end{array}$ \\
\hline ACADS e6F/R & $\begin{array}{l}\text { ggtgtcaaggcctgagctt } \\
\text { atgtccagggtttgctgtg }\end{array}$ \\
\hline ACADS e7F/R & $\begin{array}{c}\text { cagggatgggcttcaagata } \\
\text { ccacaccacaggtcagca }\end{array}$ \\
\hline ACADS e8F/R & $\begin{array}{l}\text { ggcagctgctgacctgtg } \\
\text { caggcccacacctctgac }\end{array}$ \\
\hline ACADS e9F/10R & $\begin{array}{l}\text { ggtcccctcaagggaagg } \\
\text { ggaacttgaggcacagtggt }\end{array}$ \\
\hline
\end{tabular}

ACADS - acyl-CoA dehydrogenase, C-2 to C-3 short chain

\section{Results}

Organic acids measurement in the first urine sample with GC/MS revealed increased concentration of ethylmalonic acid. Confirmation analysis for organic acids from the second urine sample showed also slightly increased methylsuccinic acid (qualitative analysis). Butirylglicine was not present (Table 2). Acylcarnitines analysis in dried blood spot

TABLE 2. Qualitative analysis of specific urine metabolites detected in samples of a patient collected on the $6^{\text {th }}$ and $13^{\text {th }}$ day of life.

\begin{tabular}{ccc}
\hline Specific metabolite & $\begin{array}{c}\text { Sampling I } \\
\mathbf{6}^{\text {th }} \text { day of life }\end{array}$ & $\begin{array}{c}\text { Sampling II } \\
\mathbf{1 3}^{\text {th }} \text { day of life }\end{array}$ \\
\hline Ethylmalonic acid & + & ++ \\
Methylsuccinic acid & - & + \\
Butyrilglycine & - & - \\
\hline
\end{tabular}

++ highly increased ; + slightly increased; - not present 
TABLE 3. Acylcarnitine analysis in dried blood spots of a patient; samples were collected on the $6^{\text {th }}$ and $13^{\text {th }}$ day of life.

\begin{tabular}{|c|c|c|c|}
\hline Acylcarnitine & $\begin{array}{c}\text { Concentration } \\
(\mu \mathrm{mol} / \mathrm{L})\end{array}$ & $\begin{array}{c}\text { Reference } \\
\text { range } \\
\text { ( } \mu \mathrm{mol} / \mathrm{L})\end{array}$ & Sampling \\
\hline $\mathrm{C} 4$ & 2.40 & $0.11-0.68$ & $6^{\text {th }}$ day of life \\
\hline $\mathrm{C} 4$ & 1.71 & $0.11-0.68$ & $13^{\text {th }}$ day of life \\
\hline
\end{tabular}

C4: C4-carnitine, butyrylcarnitine

showed increase of butyrylcarnitine, C4-carnitine. C4-carnitine was 3.5 times above the reference range $(<0.68 \mu \mathrm{mol} / \mathrm{L})$ (Table 3$)$. Acylcarnitine profile was confirmed from the second dried blood spot card. Metabolic findings were consistent with SCADD. In order to confirm the diagnosis, the DNA was isolated from peripheral blood. Sequence analysis of all exons and flanking intron sequences of ACADS gene revealed 3-bp in frame deletion at position 310-312 in homozygous state (NM_000017.2: c.310_312delGAG). The mutation results in the loss of glutamine residue at position 104 of the mature SCAD protein (p.Glu104del). The sequence variant is considered as a mutation according to MutationTaster prediction program (http://www.mutationtaster.org/). Mutation analysis of parents DNA showed the same mutation in heterozygous state. Parents are not consanguineous, but they are coming from the same community of Romani people.

\section{Discussion}

We describe a first patient of Romani origin with SCADD diagnosed in Slovenia presented with hypoglycaemia, hypotonia, seizures, failure to thrive and developmental delay. Clinical signs of a patient were consistent with the most frequent signs, reported in the literature $(3,4,16)$. Due to observed clinical signs, diagnostic tests for selective screening of metabolic disorders were done. Increase of the biochemical hallmarks, C4-carnitine and ethylmalonic acid suggested SCADD. Diagnosis was confirmed with mutational analysis and homozygous deletion of 3 nucleotides at position 310-312 was detected. This mutation was to our knowledge for the first time described in homozygous state. Corydon et al. previously described the mutation as pathogenic in one patient with hypotonia and developmental delay but in heterozygous state, the second mutation was not found. Clinical signs presented in first week of life as hypotonia and developmental delay. Fibroblast SCAD activity was undetectable (7). According to the literature, molecular effect of specific SCAD variations on cellular function is unclear (1). Since our nucleotide variation is $3 \mathrm{bp}$ deletion on both alleles we believe that it has higher impact on a clinical picture than other described mutations in the ACADS gene.

We are aware of limitations of this case report. There were scarce data on neurological evaluation of a patient and enzyme activity has not been measured. We also don't have information about parents history related with certain signs and symptoms suggestive for SCADD. Additionally, we don't have results for biochemical analyses for parents (organic acids and acylcarnitine analysis).

The disorder has been reported in infants, children and adults $(7,17)$. Most symptomatic cases of SCADD have been diagnosed due to large diagnostic work-up on children with non-specific findings such as development delay, failure to thrive and myopathy (18). However, the clinical relevance of SCADD is questionable and still under discussion. The reasons are the variability and the nature of the symptoms and signs and existence of many patients without signs of the disease that are diagnosed through newborn screening programs $(4,14,19)$. Those patients may remain asymptomatic even with the same genotype as symptomatic patients (13). Moreover, there were alternate explanations for clinical findings when present (4). The reported spectrum of clinical signs is difficult to correlate to enzymatic defect (20-22). Pederson et al. propose that SCADD should be considered as a disorder of protein folding that can lead to clinical disease in combination with other genetic and environmental factors (1). Also the need to treat affected individuals is unclear. It was shown that only patients with history of hypoglycaemia had development of hypoglycaemia during fasting test (2). Different proteomic studies search for biomarkers that will help to distinguish asymptomatic 
SCAD deficiency from individuals at risk to develop symptoms $(23,24)$ and genome-wide association studies identify genetic variants associated with relative levels of C4-carnitine (25).

The described cases of SCADD indicate that thorough workup off all patients with neurological signs like development delay, seizures and hypotonia is required. The true relationship between this enzyme deficiency and clinical outcomes will be determined only by long-term follow-up data

\section{References}

1. Pedersen $C B$, Kølvraa $S$, Kølvraa A, Stenbroen V, Kjeldsen $M$, Ensenauer $R$, et al. The ACADS gene variation spectrum in 114 patients with short-chain acyl-CoA dehydrogenase (SCAD) deficiency is dominated by missense variations leading to protein misfolding at the cellular level. Hum Genet 2008;124:43-56. http://dx.doi.org/10.1007/s00439-0080521-9.

2. Van Maldegem BT, Duran $M$, Wanders RJA, Waterham HR, de Koning TJ, Rubio E, et al. Fasting and fat-loading tests provide pathophysiological insight into short-chain acyl-coenzyme a dehydrogenase deficiency. I Pediatr 2010;156:121-7. http://dx.doi.org/10.1016/j. jpeds.2009.07.008.

3. Van Maldegem BT, Wanders RJA, Wijburg FA. Clinical aspects of short-chain acyl-CoA dehydrogenase deficiency. J Inherit Metab Dis 2010;33:507-11. http://dx.doi. org/10.1007/s10545-010-9080-z.

4. Gallant NM, Leydiker K, Tang H, Feuchtbaum L, Lorey F, Puckett $R$, et al. Biochemical, molecular, and clinical characteristics of children with short chain acyl-CoA dehydrogenase deficiency detected by newborn screening in California. Mol Genet Metab 2012;106:55-61. http://dx.doi. org/10.1016/j.ymgme.2012.02.007.

5. Amendt BA, Greene C, Sweetman L, Cloherty J, Shih V, Moon $A$, et al. Short-chain acyl-coenzyme $A$ dehydrogenase deficiency. Clinical and biochemical studies in two patients. J Clin Invest 1987;79:1303-9. http://dx.doi. org/10.1172/JCI112953.

6. Van Maldegem BT, Kloosterman SF, Janssen WJ, Augustijn $P B$, van der Lee $J H$, ljlst $L$, et al. High prevalence of shortchain acyl-CoA dehyydrogenase deficiency in the Netherlands, but no association with epilepsy of unknown origin in childhood. Neuropediatrics 2011;42:13-7. http://dx.doi. org/10.1055/s-0031-1275342.

7. Corydon MJ, Vockley J, Rinaldo P, Rhead WJ, Kjeldsen $M$, Winter $V$, et al. Role of common gene variations in the molecular pathogenesis of short-chain acyl-CoA dehydrogenase deficiency. Pediatr Res 2001;49:18-23. http://dx.doi. org/10.1203/00006450-200101000-00008. on SCADD patients diagnosed by selective screening and those diagnosed by newborn screening.

In our case clinical picture of a patient, biochemical parameters and genetic data were concordant and showed definitely SCAD deficiency. We hope that this will contribute to the better understanding of this disease.

\section{Potential conflict of interest}

None declared.

8. Bok LA, Vreken $P$, Wijburg FA, Wanders RJA, Gregersen $N$ Corydon MJ, et al. Short-chain Acyl-CoA dehydrogenase deficiency: studies in a large family adding to the complexity of the disorder. Pediatrics 2003;112:1152-5. http:// dx.doi.org/10.1542/peds.112.5.1152.

9. Pedersen $C B$, Bross P, Winter VS, Corydon TJ, Bolund L, Bartlett $K$, et al. Misfolding, degradation, and aggregation of variant proteins. The molecular pathogenesis of short chain acyl-CoA dehydrogenase (SCAD) deficiency. J Biol Chem 2003;278:47449-58. http://dx.doi.org/10.1074/jbc. M309514200.

10. Tein I, Elpeleg O, Ben-Zeev B, Korman SH, Lossos A, Lev D, et al. Short-chain acyl-COA dehydrogenase gene mutation $(c .319 C>T)$ presents with clinical heterogeneity and is candidate founder mutation in individuals of Ashkenazi Jewish origin. Mol Genet Metab 2008;93:179-89. http:// dx.doi.org/10.1016/j.ymgme.2007.09.021.

11. Pena $L$, Angle B, Burton B, Charrow J. Follow-up of patients with short-chain acyl-CoA dehydrogenase and isobutyryl-CoA dehydrogenase deficiencies identified through newborn screening: one center's experience. Genet Med Off J Am Coll Med Genet 2012;14:342-7. http://dx.doi. org/10.1038/gim.2011.9.

12. Barth $M$, Ottolenghi $C$, Hubert $L$, Chrétien D, Serre V, Gobin $S$, et al. Multiple sources of metabolic disturbance in ETHE1-related ethylmalonic encephalopathy. J Inherit Metab Dis 2010;33:S443-53. http://dx.doi.org/10.1007/s10545010-9227-y.

13. Kim SH, Park HD, Sohn YB, Park SW, Cho SY, Ji S, et al. Mutations of ACADS gene associated with short-chain acylcoenzyme A dehydrogenase deficiency. Ann Clin Lab Sci 2011;41:84-8.

14. Waisbren SE, Levy $H L$, Noble M, Matern $D$, Gregersen $N, P a$ sley $K$, et al. Short-chain acyl-CoA dehydrogenase (SCAD) deficiency: an examination of the medical and neurodevelopmental characteristics of 14 cases identified through newborn screening or clinical symptoms. Mol Genet Metab 2008;95:39-45. http://dx.doi.org/10.1016/j. ymgme.2008.06.002. 
15. Van Maldegem BT, Waterham HR, Duran $M$, van der Vlies $M$, van Woerden CS, Bobu LL, et al. The 625G>A SCAD gene variant is common but not associated with increased C4-carnitine in newborn blood spots. J Inherit Metab Dis 2005;28:55762. $h$ ttp://dx.doi.org/10.1007/s10545-005-0557-0.

16. Jethva R, Bennett MJ, Vockley J. Short-chain acyl-coenzyme A dehydrogenase deficiency. Mol Genet Metab 2008;95:195200. http://dx.doi.org/10.1016/j.ymgme.2008.09.007.

17. Bhala A, Willi SM, Rinaldo P, Bennett MJ, Schmidt-Sommerfeld $E$, Hale DE. Clinical and biochemical characterization of short-chain acyl-coenzyme A dehydrogenase deficiency. J Pediatr 1995;126:910-5. http://dx.doi.org/10.1016/ S0022-3476(95)70207-5.

18. Reddy GS, Sujatha M. A Rare Case of Short-Chain Acyl-COA Dehydrogenase Deficiency: The Apparent Rarity of the Disorder Results in Under Diagnosis. Indian J Clin Biochem IJCB 2011;26:312-5. http://dx.doi.org/10.1007/s12291011-0139-x.

19. Cajaiba MM, Hughes AMS, Pierson CR. Central nervous system pathology in an infant with short-chain acyl-CoA dehydrogenase deficiency (SCADD): evidence for abnormal neuronal migration as part of its phenotypic spectrum. Clin Neuropathol 2012;31:386-8. http://dx.doi.org/10.5414/ NP300473.

20. Gregersen N, Andresen BS, Bross P. Prevalent mutation in fatty acid oxidation disorders: diagnostic considerations. Eur J Pediatr 2000;159:S213-18. http://dx.doi.org/10.1007/ PL00014406.
21. Gregersen $N$, Bross $P$, Andresen BS. Genetic defects in fatty acid beta-oxidation and acyl-CoA dehydrogenases. Eur J Biochem 2004;271:470-82. http://dx.doi.org/10.1046/ j.1432-1033.2003.03949.x.

22. Van Maldegem BT, Duran $M$, Wanders RJ, Niezen-Koning $K E$, Hogeveen $M$, ljlst $L$, et al. Clinical, biochemical, and genetic heterogeneity in short-shain acyl-coenzyme $A$ dehydrogenase deficiency. JAMA 2006;296:943-52. http:// dx.doi.org/10.1001/jama.296.8.943.

23. Wang W, Mohsen AW, Uechi G, Schreiber E, Balasubramani $M$, Day $B$, et al. Complex changes in the liver mitochondrial proteome of short chain acyl-CoA dehydrogenase deficient mice. Mol Gen Metab 2014;112:30-9. http://dx.doi. org/10.1016/j.ymgme.2014.02.014.

24. Edhager AV, Stenbroen $V$, Nielsen NS, Bross $P$, Olsen RK, Gregersen $N$, et al. Proteomic investigation of cultivated fibroblasts from patients with mitochondrial shortchain acyl-CoA dehydrogenase deficiency. Mol Genet Metab 2014;111:360-8. http://dx.doi.org/10.1016/j. ymgme.2014.01.007.

25. Ryckman KK, Smith CJ, Jelliffe-Pawlowski LL, Momany AM, Berberich SL, Murray JC. Metabolic heritability at birth: implications for chronic disease research. Hum Genet 2014;133:1049-57. http://dx.doi.org/10.1007/s00439-014$1450-4$ 